ARTICLE

DOI: $10.1038 / s 41467-018-03259-z$

OPEN

\title{
Molecular demultiplexer as a terminator automaton
}

\author{
Ilke S. Turan1, Gurcan Gunaydin², Seylan Ayan³ \& Engin U. Akkaya (1) 1,3
}

Molecular logic gates are expected to play an important role on the way to information processing therapeutic agents, especially considering the wide variety of physical and chemical responses that they can elicit in response to the inputs applied. Here, we show that a 1:2 demultiplexer based on a $\mathrm{Zn}^{2+}$-terpyridine-Bodipy conjugate with a quenched fluorescent emission, is efficient in photosensitized singlet oxygen generation as inferred from trap compound experiments and cell culture data. However, once the singlet oxygen generated by photosensitization triggers apoptotic response, the $\mathrm{Zn}^{2+}$ complex then interacts with the exposed phosphatidylserine lipids in the external leaflet of the membrane bilayer, autonomously switching off singlet oxygen generation, and simultaneously switching on a bright emission response. This is the confirmatory signal of the cancer cell death by the action of molecular automaton and the confinement of unintended damage by excessive singlet oxygen production.

\footnotetext{
${ }^{1}$ UNAM-National Nanotechnology Research Center, Bilkent University, 06800 Ankara, Turkey. ${ }^{2}$ Department of Basic Oncology, Hacettepe University, 06100 Ankara, Turkey. ${ }^{3}$ Department of Chemistry, Bilkent University, 06800 Ankara, Turkey. Ilke S. Turan and Gurcan Gunaydin contributed equally to this work. Correspondence and requests for materials should be addressed to E.U.A. (email: eua@fen.bilkent.edu.tr)
} 
M ore than two decades after the seminal work by de Silva et al. ${ }^{1}$, molecule-based logic gates have reached a level of considerable sophistication ${ }^{2-4}$. Many examples of basic Boolean logic gates and various implementations of both combinatorial and sequential logic were reported ${ }^{5-10}$. While it is clear that more advanced digital designs may require novel integration mechanisms of chemical logic gates, functional equivalents of more complex information processing is still possible with simple molecules or molecular assemblies as a result of their unique characteristics. However, despite this impressive progress, the power of chemical and molecular logic gates beyond the exploratory phase is yet to be convincingly demonstrated, ${ }^{11}$ with what is colloquially referred to as a killer app $p^{12}$.

The most likely field where molecule-based information processing agents would find a niche for true utility is therapeutic medicine. We previously described various protocols to combine molecular logic gate notions with photosensitized generation of short-lived cytotoxic species, singlet oxygen ${ }^{3,13,14}$. Photosensitized generation of singlet oxygen in tumors, in or around cancer cells, is the essence of photodynamic therapy of cancer ${ }^{15-18}$. The fate of the singlet excited state $\left(S_{1}\right)$ of the photosensitizer is strongly related to the relative efficiencies and rates of photophysical processes involved. Among these, the most relevant are radiative transition from $\mathrm{S}_{1}$ to $\mathrm{S}_{0}$ (fluorescence) and intersystem crossing to $\mathrm{T}_{1}$ triplet state (isc). Efficiency of access to the triplet manifold is directly linked to the singlet oxygen quantum yield. Thus, considering the fact that in the photosensitization process, fluorescence and intersystem crossing (hence, singlet oxygen generation) are mutually antagonistic, and at least in principle, it should be possible to switch between these two processes. The digital equivalent is that of a demultiplexer (DEMUX) circuit ${ }^{19}$, which takes single data input, and uses $n$ select/address inputs to switch between $2^{n}$ possible outputs (Fig. 1).
Chemical implementation of this idea relies on the photophysics of the meso-pyridyl (or oligopyridyl) substituted Bodipy dyes (Fig. 1d). Bodipy dyes are exceptionally versatile and chemically malleable chromophores. The reference Bodipy chromophore (B-1) has high fluorescence quantum yield with a small Stokes' shift and, on excitation, would have negligible access to the triplet manifold. This is also in part the reason for higher chemical stability of this class of dyes under ambient conditions. In our earlier investigations ${ }^{20}$ aiming cation-responsive fluorophores (chemosensors), we observed a sharp decrease in the emission of bipyridyl-Bodipy compound (B-2) when an acid (trifluoroacetic acid (TFA)) or zinc perchlorate was added. Apparently, protonation of the ligand, or complexation with a +2 charged cation, transforms the ligand into a more easily reducible species, and photoinduced electron transfer from the excited Bodipy dye to the bipyridyl ligand becomes more efficient. Resulting charge transfer state (CTS) is responsible for the overall non-radiative relaxation (hence quenching). The orthogonal arrangement of the ligand and the chromophore is expected to facilitate intersystem crossing to the triplet state, as the charge recombination is typically accompanied with the population of the triplet excited state ${ }^{21}$. Considering recent data on the photophysics of the similar Bodipy-pyridyl ligand systems ${ }^{22}$, we concluded that such protonated pyridinium, or quaternized pyridinium substituted Bodipy dyes, and the $\mathrm{Zn}^{2+}$ complexes are likely to be more efficient generators of singlet oxygen compared to the parent compounds from which they are derived due to enhanced yield of the triplet state. Another useful aspect of this change is that it is chemically reversible; i.e, if the protonation is reversed, or charge on the $\mathrm{Zn}^{2+}$ ion is neutralized (even partially), a return to the original state of affairs should be expected. Thus, the addition of phosphate to the $\mathbf{B}-2 / \mathrm{Zn}^{2+}$ complex destabilizes the CT state and restores the fluorescence emission intensity. a

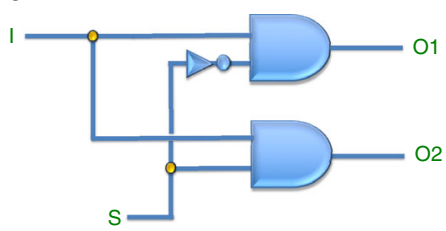

b

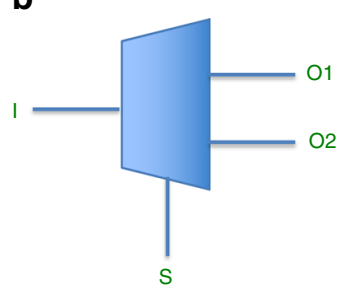

C

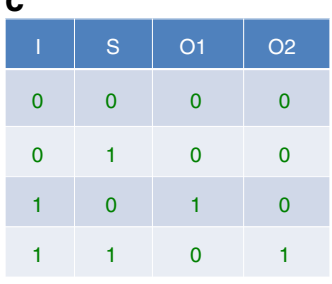

d

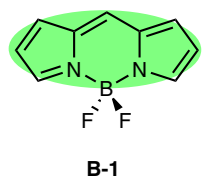

B-1

$\Phi_{\text {isc }}=0.0011$

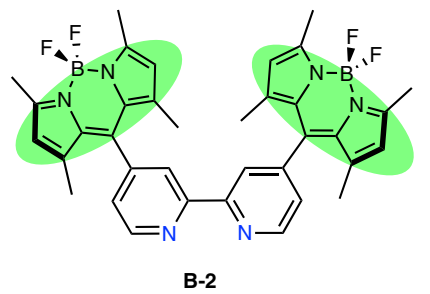

B-2

$$
\begin{gathered}
\Phi_{\mathrm{fl}}=0.39 \\
\downarrow+\mathrm{Zn}^{2+} \text { or } \mathrm{H}^{+} \\
\Phi_{\mathrm{fl}}<0.002
\end{gathered}
$$

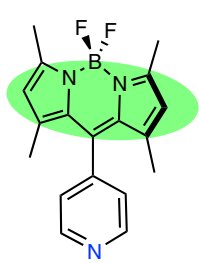

B-3

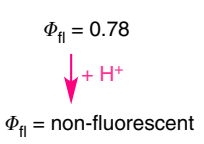

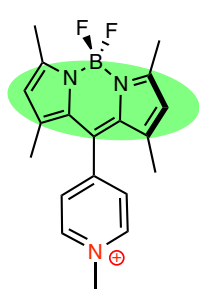

B-4

$\Phi_{\mathrm{fl}}=0.005$

$\Phi_{\text {isc }}=0.75$

Fig. 1 DEMUX combinatorial circuit and structures of relevant Bodipy compounds. a DEMUX circuit with standard logic gate symbols. b Isosceles trapezoid is a common symbol for the MUX/DEMUX circuits. I is the data input, $\mathrm{S}$ is the select or address input, and $\mathrm{O} 1$ and $\mathrm{O} 2$ are two different outputs. c The truth

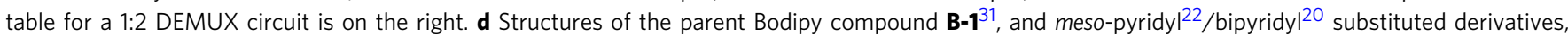
together with their respective fluorescence and intersystem crossing quantum efficiencies are demonstrated. Protonation of both B-2 and B-3 result in the pyridinium cation, which switches on the intramolecular charge transfer process. Coordination of $\mathrm{Zn}^{2+}$ ions or quaternization at the pyridine nitrogen (B-4) results in the same photophysical consequences 
In a demultiplexer, the select input would determine the choice between the possible outputs. Searching for a candidate as the select input, we looked into the possibility of making use of structural changes taking place in the cell membranes during apoptosis. Fluorescence imaging of apoptosis relies on the loss of membrane asymmetry (adenosine triphosphate (ATP)-dependent enzyme called flippase normally keeps phosphatidylserine inside the cell) $)^{23,24}$ as the negatively charged lipids and particularly phosphatidylserine is flipped to the extracellular side of the bilayer. The change is mediated by enzymes such as scramblase, which exposes phosphatidylserine on the cell's surface without consuming ATP 23,25 . A selective probe for this event is the protein Annexin V, which selectively binds to phosphatidylserine. Since Annexin V can be conveniently labeled with fluorescent dyes of different emission colors, it provides a set of useful tools for detecting apoptotic cells. There is also an interest in finding simpler, non-protein reporters of apoptosis, and in most of the reported examples ${ }^{26}$, the part which interacts with the phosphatidylserine unit is a $\mathrm{Zn}^{2+}$ complex, in which the metal ion is held in place by pyridine-derived ligands, presenting coordination sites to the anionic groups of the phosphatidylserine ${ }^{27}$.

In this work, we present a unique molecular device based on above considerations. The molecular automaton described here initially generates singlet oxygen to trigger apoptosis in cancer cells, and then, in response to apoptotic changes in the membrane structure, shuts off singlet oxygen generation and produces emission as a result of its interaction with the exposed phosphatidylserines in the outer leaflet of the apoptotic cell membranes. This could be interpreted as the response of a molecular demultiplexer which takes light as an input and phosphatidylserines as the switch. The two alternative outputs are singlet oxygen and light.

\section{Results}

Operation of the automaton. The structures of the targeted compounds T-1 and T-2 for synthesis are shown in Fig. 2 a.
Terpyridyl-Bodipy compounds T-1 and T-2 differ only in the groups attached to the boron bridge. While compound T-2 is appropriate for chemical characterization, compound $\mathbf{T}-\mathbf{1}$ is better for biological media due to additional solubilizing oligoethyleneglycol units. The use of terpyridine instead of bipyridine ligand is favored due to the stronger affinity of the former ligand to $\mathrm{Zn}^{2+}$ ions in aqueous media. The changes in emission were studied in acetonitrile. A $2.0 \mu \mathrm{M}$ solution of the model DEMUX T-2 has an intense emission band $\left(\phi_{\mathrm{F}}=0.34\right)$ with a maximum at $517 \mathrm{~nm}$. Titration with $\mathrm{Zn}^{2+}$ ions in the form of perchlorate salt results in sharp loss of emission intensity, with emission band moving to longer wavelengths, as the CT component in the emission becomes more prominent.

However, the addition of tetrabutylammonium phosphate completely reverses this change at $60 \mu \mathrm{M}$ concentration in acetonitrile. We then studied singlet oxygen generation rates under the same conditions for $+/-$ phosphate: the singlet oxygen trap used is 1,3-diphenylisobenzofuran, its absorbance at $410 \mathrm{~nm}$ decreases due to a [4+2] cycloaddition with singlet oxygen, which is followed by decomposition. In accordance with our design expectations, when irradiated with a $\lambda=522$ $\mathrm{nm}$ light-emitting diode array (at $98.0 \mu \mathrm{mol} \mathrm{m}^{-2} \mathrm{~s}^{-1}$ photon flux), the Zinc ${ }^{2+}$ complex is quite an effective photosensitizer. The singlet oxygen quantum yield $\left(\phi_{\Delta}\right)$ was determined in reference to Eosin $\mathrm{Y}$, and found to be 0.11 . However, the addition of $2.0 \mathrm{mM}$ phosphate in the form of tetrabutylammonium phosphate essentially stops singlet oxygen generation (Fig. 3f). Thus, it is clear that fluorescence emission intensity and the singlet oxygen generation efficiency are inversely coupled, and the switch is the phosphate ions in solution. Operation of the molecular DEMUX circuit based on compound T-2 was confirmed. We also determined the singlet oxygen quantum yield of T-1 as 0.10 .

Once the chemical validation was obtained, we ventured into cell culture experiments. Cell culture assays were performed with a human cancer suspension cell line-chronic myelogenous
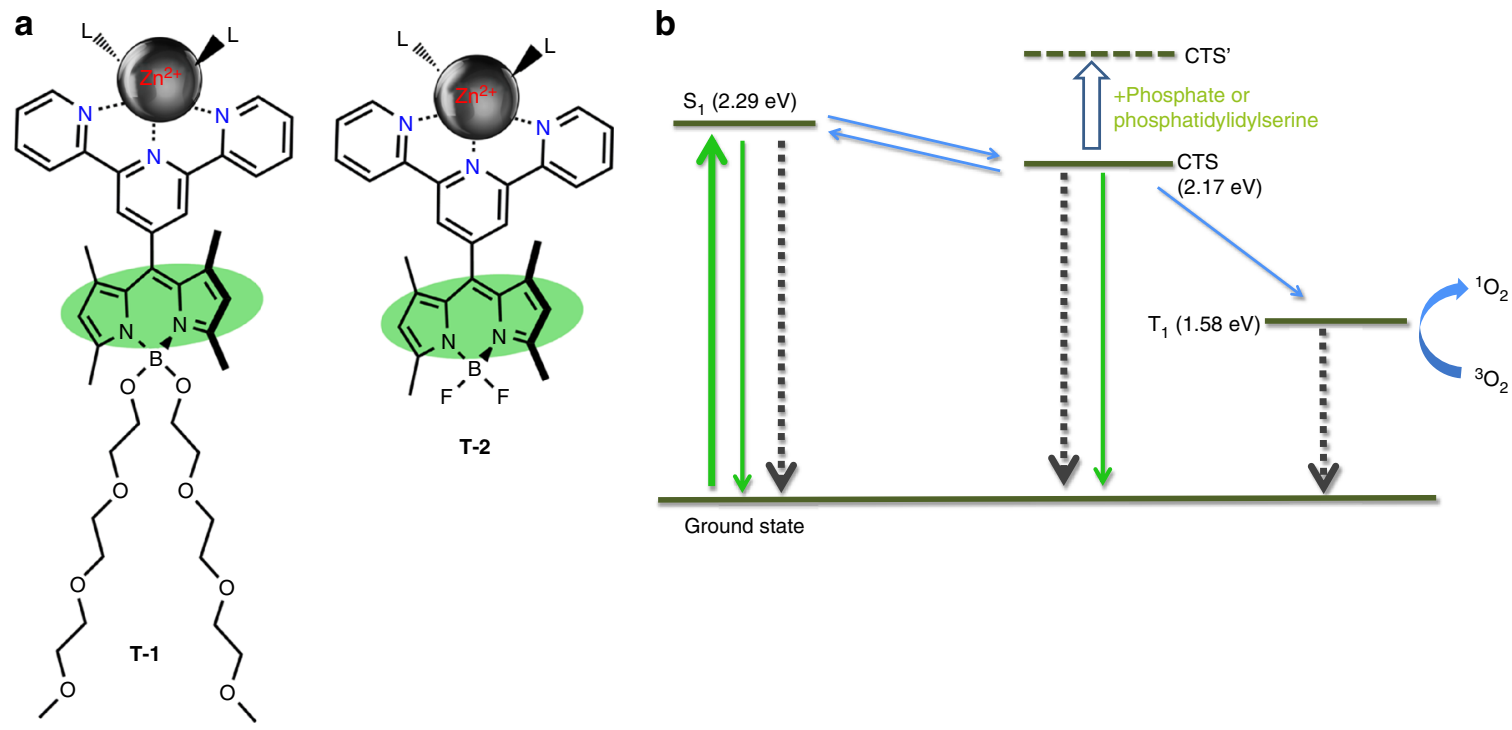

Fig. 2 The structures of the molecular automata $\mathbf{T - 1}$ and $\mathbf{T - 2}$ and the Jablonski diagram depicting processes involved. a $L$ indicates solvent molecules as ligands, water, or acetonitrile. Bodipy and the terpyridyl planes are orthogonal to each other due to the presence of 1,7-dimethyl substitution of the Bodipy chromophore. Double mTEG (methoxytriethyleneglycol) substitution at the boron center enhances water solubility. b Thicker green arrow is absorption, the other green arrows indicate radiative relaxations. Black-dashed arrows are non-radiative relaxation processes. Blue arrows indicate transitions between various excited states. CTS to $\mathrm{T}_{1}$ transition is particularly enhanced because of the orthogonal geometry of the terpyridyl-Bodipy diad. Energy levels were experimentally estimated using the spectral data $\left(\mathrm{S}_{1}, \mathrm{CTS}\right)$ in analogy to previous literature ${ }^{22}$, or based on the phosphorescence data ${ }^{32}$ for similar Bodipy compounds $\left(\mathrm{T}_{1}\right)$ 
a
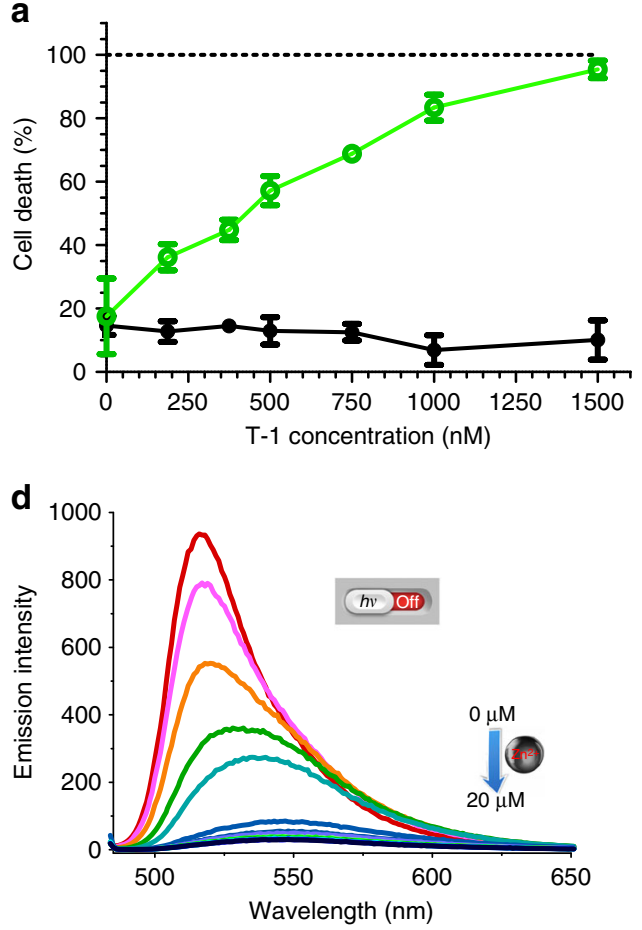

b

\begin{tabular}{|c|c|c|c|}
\hline $\begin{array}{c}\text { Input } \\
h v \\
(\mathrm{exc})\end{array}$ & Switch & Out1 & Out2 \\
\hline 0 & 0 & 0 & $\begin{array}{c}h v \\
(\mathrm{em})\end{array}$ \\
\hline 0 & 1 & 0 & 0 \\
\hline 1 & 0 & 1 & 0 \\
\hline 1 & 1 & 0 & 1 \\
\hline
\end{tabular}

C

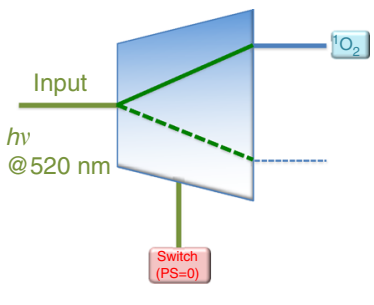

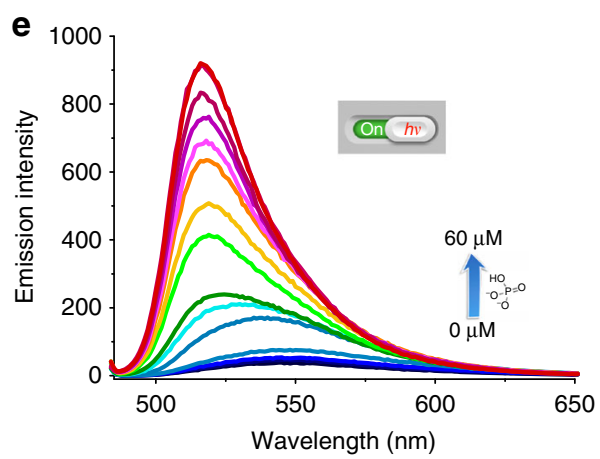

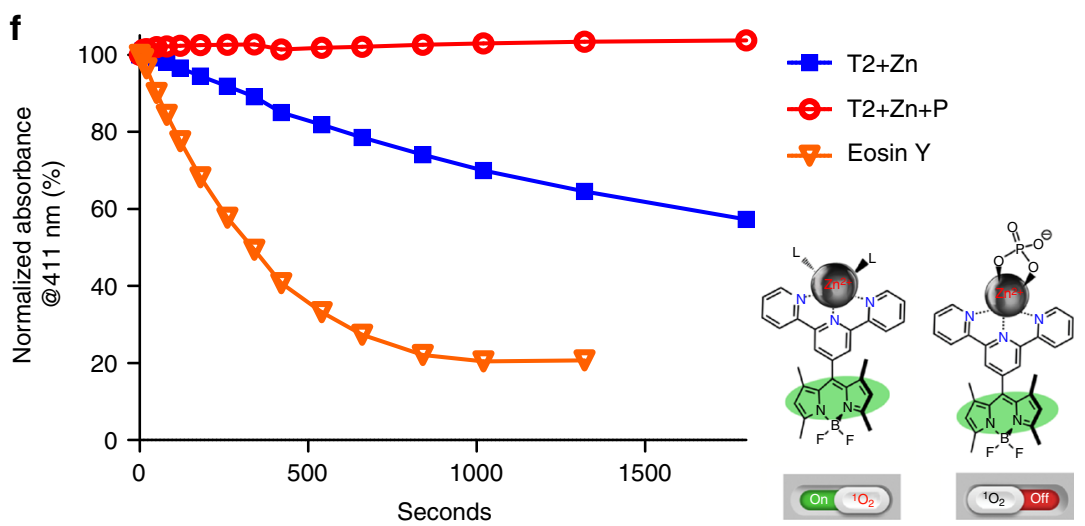

Fig. 3 Operation of the molecular automata as evidenced by spectroscopic and cell culture data. a MTT assay data: Green open circles correspond to percent death of $\mathrm{K} 562$ cells under irradiation at $522 \mathrm{~nm}$ for $12 \mathrm{~h}$ followed by continued incubation in dark for another $12 \mathrm{~h}$. Solid black circles correspond to cells kept under identical conditions of incubation with the agent, but in dark. Positive control (dashes at $100 \%$ line) corresponds to cells incubated in DMSO-growth medium mixture $(50 / 50, v / v)$. b The truth table with the data and switch inputs, and the corresponding outputs clearly identified, and the particular set of conditions valid under the light irradiation conditions on power-up were highlighted. c Switch input 0 (no added phosphate in the model system or lack of phosphatidylserine (PS) in the external leaflet of the cell membrane in the cell cultures) selects singlet oxygen as the primary output. $\mathbf{d}$ The model compound $\mathbf{T - 2}$, which is the $\mathrm{Zn}^{2+}$ complex of the meso-terpyridyl-bodipy compound, has a very low fluorescence emission intensity in acetonitrile. $\mathbf{e}$ The addition of phosphate ions results in a very sharp increase in emission intensity. The low emission intensity is due to the availability of a charge transfer state (CTS) resulting in enhanced intersystem crossing, which in turn leads to efficient generation of singlet oxygen. $\mathbf{f}$ The decrease in the absorbance at $411 \mathrm{~nm}$, in an oxygen saturated ethanol solution of selective singlet oxygen trap DPBF $(50 \mu \mathrm{M})$ in the presence of $2.0 \mu \mathrm{M}$ T-2 and under irradiation with $522 \mathrm{~nm}$ green LED light source (solid blue squares). The singlet oxygen quantum yield $\left(\phi_{\Delta}\right)$ of $\mathbf{T - 2}$ is 0.11 . The addition of phosphate (red open circles) destabilizes the CTS state, blocking access to the triplet manifold. The absorbance data presented is the net absorbance values obtained by subtracting any background decrease in the probe absorbance due to light alone

leukemia (K562). The cells were incubated with Dulbecco's modified Eagle's medium (DMEM) supplemented with $20 \%$ fetal bovine serum at the environmental conditions of $37^{\circ} \mathrm{C}, 5 \%$ $\mathrm{CO}_{2}$, and $60 \%$ humidity. Cells were treated with varying concentrations of the molecular automaton T-1 $(187.5 \mathrm{nM}-1.5$ $\mu \mathrm{M})$ and illuminated with a green light source $(\lambda=522 \mathrm{~nm}$ light-emitting diode array, $98.0 \mu \mathrm{mol} \mathrm{m} \mathrm{m}^{-2} \mathrm{~s}^{-1}$ photon flux) for a continuous duration of $12 \mathrm{~h}$. This $12 \mathrm{~h}$ period of illumination was followed by $12 \mathrm{~h}$ of incubation in the dark (total $24 \mathrm{~h}$ ). The control group of the cells were incubated in the dark, for the exact duration of $24 \mathrm{~h}$ under identical environmental conditions. The MTT (3-(4, 5-dimethylthiazolyl-2)-2,5-diphenyltetrazolium bromide) assay was used in order to assess cell viability and cytotoxicity. Even the low doses of the automaton T-1 seem to have resulted in a significant decrease of the cell viability (Fig. 3a, points and error bars designate means and 
standard deviations, respectively). The $\mathrm{CC}_{50}$ (50\% cytotoxic concentration) value of the T-1 subjected to green light was estimated by fitting a model with non-linear regression (approximately $365 \mathrm{nM}$; the $\mathrm{CC}_{50}$ value of the compound in the dark condition cannot be estimated since its projection is clearly out of the scale of the model fit).

Flow cytometry and microscopy. In order to confirm the MTT assay results, and the switch from singlet oxygen generation to the emission/signaling mode; Annexin V detection protocol for apoptosis was performed using flow cytometry. The percentage of fluorescent-labeled Annexin V (phycoerythrin (PE))-stained cells was much higher in the illuminated cell population compared with those incubated in the dark (73.7 vs. $18.0 \%$; Fig. 4a, blue shaded areas represent the cells in the dark, green shaded areas represent the irradiated cells). In addition, $71.7 \%$ of irradiated K562 cells (Fig. 4b) were positive for T-1 in contrast to $18.0 \%$ of the cells incubated in the dark (Fig. 4c), demonstrating that
Annexin $\mathrm{V}$ and the automaton targets the same kind of cell membranes with outer leaflet enriched in phosphatidylserine, which are undergoing apoptosis. Cancer cells, which are not killed, or not undergoing apoptosis, are not marked by the agent, but subjected to cytotoxic singlet oxygen attack, when the automaton is powered up by irradiation. Photocytotoxicity was further revealed in a 2-color analysis with PE-Annexin V in order to specifically target and identify apoptotic cells in conjunction with the T-1 (Fig. 4d). Approximately $70 \%$ of the illuminated cells were positive for both PE-Annexin V and T-1 and about $16 \%$ of the cells were negative for both (area A++ and A-- in Fig. 4d, respectively), confirming that the terminator automaton turns on the emission signal only when the cells are undergoing apoptotic death process.

Microscopy. Confocal microscopy provided further evidence corroborating the cytotoxicity results from MTT assays and flow cytometry, as well as the co-staining of PE-Annexin V and T-1 in

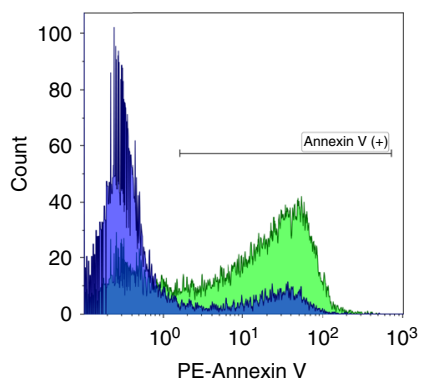

d

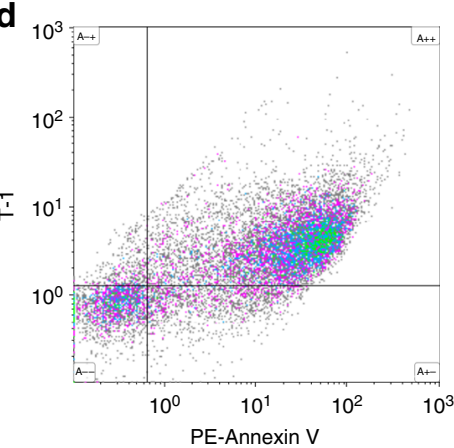

g

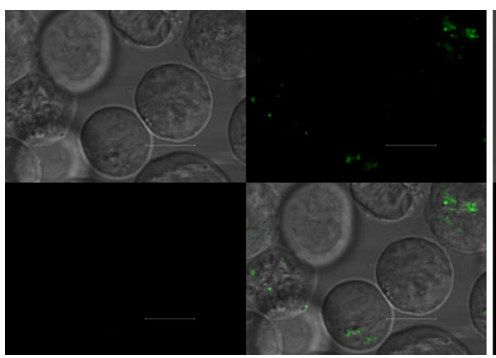

b

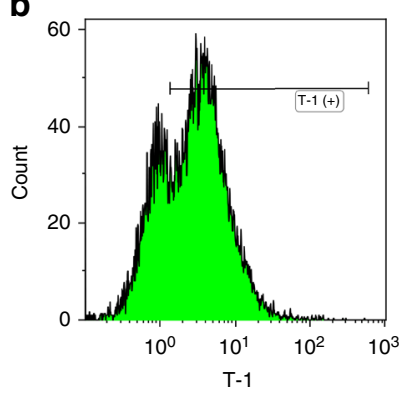

e

\begin{tabular}{|cccc}
$\begin{array}{c}\text { Input } \\
h v \\
(\mathrm{exc})\end{array}$ & PS & $1^{1} \mathrm{O}_{2}$ & $\begin{array}{c}h v \\
(\mathrm{em})\end{array}$ \\
\hline 0 & 0 & 0 & 0 \\
\hline 0 & 1 & 0 & 0 \\
1 & 0 & 1 & 0 \\
\hline 1 & 1 & 0 & 1 \\
\hline
\end{tabular}

C

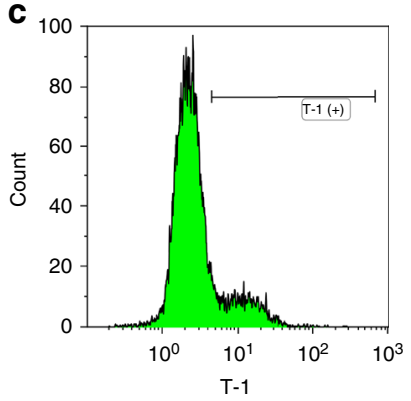

$\mathbf{f}$

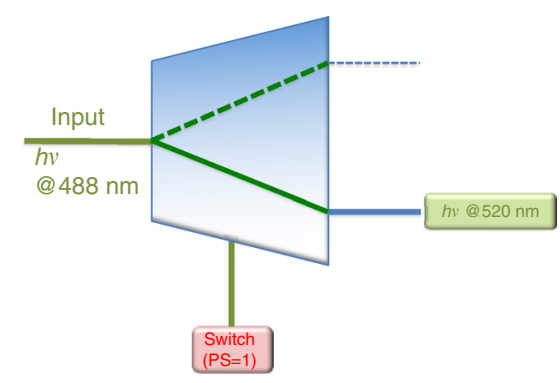

h

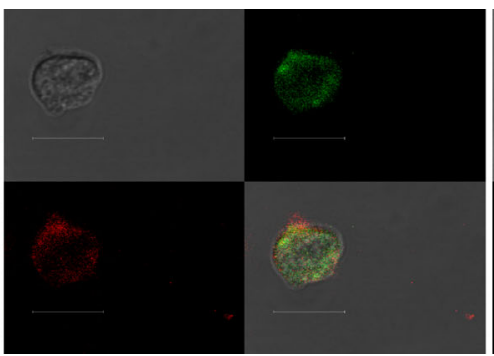

i

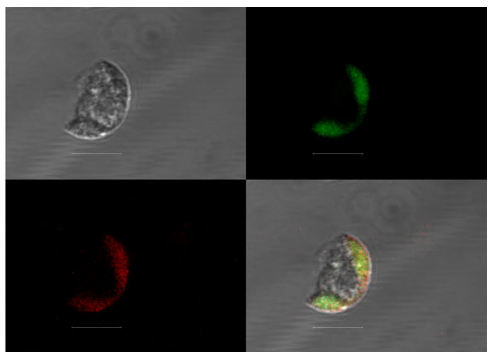

Fig. 4 T-1 signals apoptosis by switching to diagnostic mode. a PE-Annexin labels most of the cells incubated with T-1 under light irradiation (Annexin $\mathrm{V}$ (+) region of the green area). $\mathbf{b}$ Green channel: cells incubated with T-1 under irradiation. c Green channel: cells incubated with T-1 in the dark. $\mathbf{d}$ The $2 \mathrm{D}$ plot for both green and red channels: T-1 and PE-Annexin V (a specific apoptosis marker) stain the same kind of cells with a large (86\%) agreement: 70\% of the irradiated cells were co-stained with both $\mathbf{T}-\mathbf{1}$ and PE-Annexin V, indicating only apoptotic cells are fluorescently labeled with T-1. e The truth table with input, the switch and the outputs clearly identified, and the particular set of conditions were highlighted. f Switch input (appearance of phosphatidylserine in the external leaflet of the cell membrane) selects fluorescence emission as the primary output. $\mathbf{g}$ Cells treated with PE-Annexin $V$ and the $\mathbf{T - 1}$, and kept in dark, show no signs of morphological change, and the cellular membranes are not stained with either one of the agents. $\mathbf{h}$, $\mathbf{i}$ Cells were incubated with T-1, irradiated with the LED light source, then treated with PE-Annexin $\mathrm{V}$. The two agents ( $\mathbf{T}-\mathbf{1}$ and PE-Annexin $\mathrm{V}$, green and red, respectively) label the same regions in the cells undergoing apoptosis. (scale bar, $10 \mu \mathrm{m}$ ) 


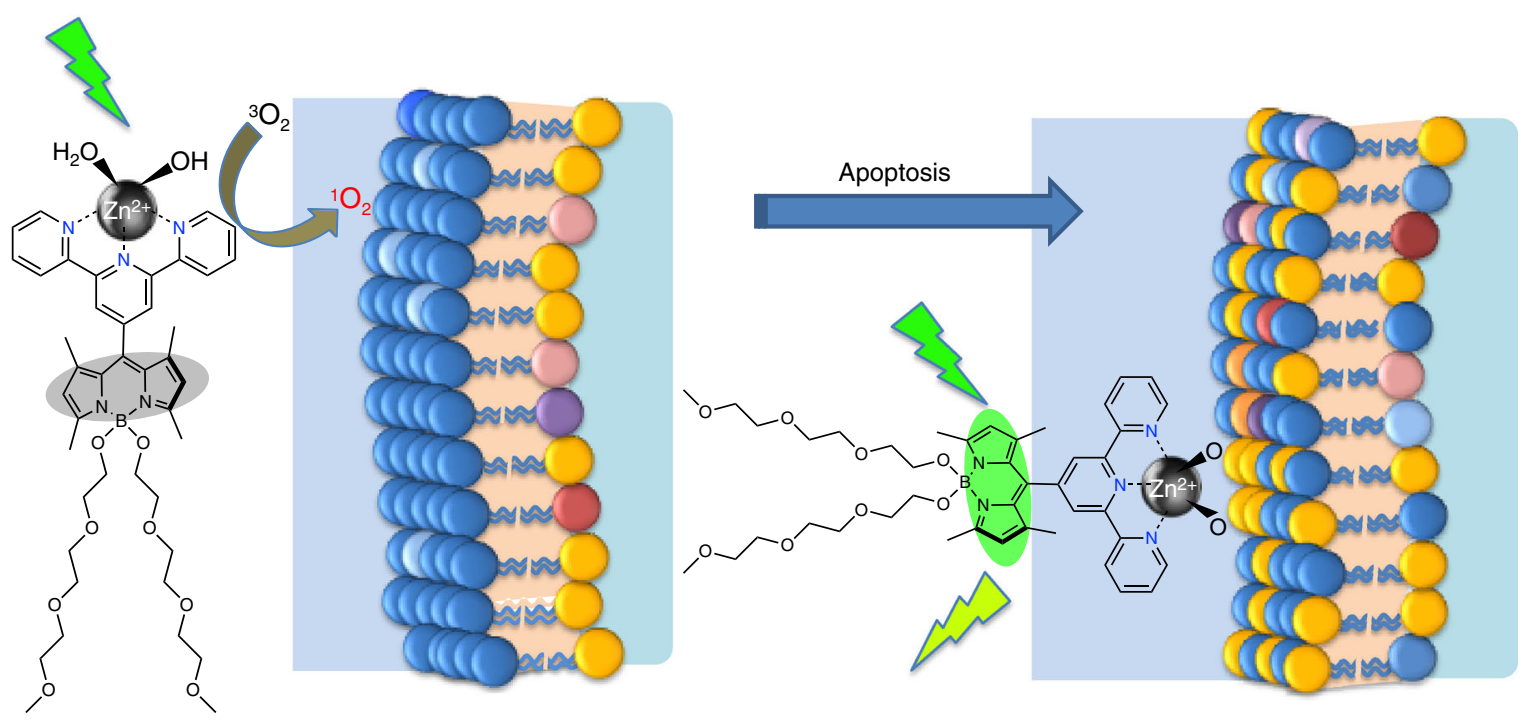

Fig. 5 T-1 induces apoptosis and then switches to diagnostic mode and fluorescently tags apoptotic cells. Blue polar heads represent phosphatidylcholine and sphingomyelins, whereas yellow, pink and purple heads represent phosphatidylserine, phosphatidylinositol and other negatively charged lipids. Apoptosis is accompanied by a loss of membrane asymmetry

flow cytometry analyses. The data demonstrate that just like PEAnnexin V, the automaton shows fluorescence signal only when attached to apoptotic cell membranes. Cellular membranes of the illuminated cells incubated with T-1 were shown to be stained with both Annexin V and T-1 $(1.5 \mu \mathrm{M})$; in contrast to their counterparts incubated in the dark, which were not stained by either T-1 $(1.5 \mu \mathrm{M})$ or PE-Annexin V. Cell membranes of the illuminated cells in the presence of $\mathbf{T}-\mathbf{1}$ appear bright green when excited at $488 \mathrm{~nm}$ and bright red when excited at $543 \mathrm{~nm}$. However, their counterparts, which had been incubated in the dark, were negative for either green or red emission. Figure $4 \mathrm{~g}-\mathrm{i}$ shows the differential interference contrast (DIC) image, T-1, Annexin $\mathrm{V}$ and merged images, respectively.

A graphical representation of the operation of the automaton T-1 is shown in Fig. 5.

\section{Discussion}

It appears that judiciously designed molecular logic devices can carry out critical information processing in or around the cells. Widely speculated nanorobots are not expected to be miniaturized versions of their macroscopic counterparts, but as illustrated here, most likely to be developed by careful control of photophysics and chemical reactivity at the individual molecule level, resulting in molecular entities with intelligent responses and actions. The zinc complex of the terpyridyl-bodipy, T-1, is approximately $3 \mathrm{~nm}$ at the longest dimension, yet it is capable of killing cancer cells when powered up by light, and then autonomously switching to a signaling mode in response to changing membrane characteristics, thus confirming apoptosis by a strong emission signal. As evidenced by the spectroscopic experiments with T-2, and flow cytometry data obtained with T-1, emission intensity and the singlet oxygen production is inversely coupled, and the fact that singlet oxygen generation is turned off once the cells show signs of apoptosis is very valuable in containing unintended damage by singlet oxygen. This clearly qualifies T-1 as a molecular terminator automaton, or an example of a functional molecular robot. While most nano-sized robotics work is at present focussed on mobility, it is interesting to note that mobility is far from being the number one issue for a functional, molecule- sized robot. Most infectious microorganisms, cells fighting against these agents, and drug molecules are efficiently carried passively via the circulatory system to any part of the body. It is the intelligent autonomous operation that provides the most crucial challenge, and the terminator automaton T-1 demonstrates one way of moving ahead to meet that challenge.

\section{Methods}

Cell culture and MTT assay. K562 human chronic myelogenous leukemia suspension cells were cultured in $25 \mathrm{~cm}^{2}$ culture flasks containing DMEM (Gibco, 11971-025) supplemented with $20 \%$ fetal bovine serum in a cell culture incubator at $37^{\circ} \mathrm{C}, 5 \% \mathrm{CO}_{2}$, and $60 \%$ humidity. The main functional goals of the current study are to study the cytotoxicity of T-1 under illumination and its staining ability of the apoptotic cells. Since phosphate in the growth media can interfere with experimental results, we used DMEM w/o phosphate supplemented with $20 \%$ fetal bovine serum that has been dialyzed extensively at $4{ }^{\circ} \mathrm{C}$ against isotonic saline $(0.15 \mathrm{M} \mathrm{NaCl})$ using dialysis tubing (Sigma-Aldrich, USA (D7884)), since the viability of the cells cultured in such conditions is known to be not hampered for periods even more than $24 \mathrm{~h}^{28}$. This cell culture medium was also utilized for analyses with flow cytometry and confocal laser scanning microscopy to study cytotoxicity and cellular staining.

The compound was diluted in cell culture medium and assay concentrations were freshly prepared. Cell viability/death was evaluated by MTT assay. Briefly, 50 $\mu \mathrm{l}$ cell suspensions in culture medium containing $3 \times 10^{4} \mathrm{~K} 562$ cells were plated in 96-well flat-bottom culture plates (Corning, MA, USA) and incubated for $12 \mathrm{~h}$ to recover from handling. Varying concentrations of the chemical compound in cell culture medium were added into each well (the final concentrations were 187.5 $\mathrm{nM}-1.5 \mu \mathrm{M})$ in quadruplicate. The experimental group of the cells were illuminated with a green light source $(\lambda=522 \mathrm{~nm}$ light-emitting diode array, 98 $\mu \mathrm{mol} \mathrm{m} \mathrm{m}^{-2} \mathrm{~s}^{-1}$ photon flux, distance between light source and cells: $10 \mathrm{~cm}$ ) for a continuous duration of exactly $12 \mathrm{~h}$ in a culture incubator $\left(37^{\circ} \mathrm{C}, 5 \% \mathrm{CO}_{2}, 60 \%\right.$ humidity). This $12 \mathrm{~h}$ period of illumination was followed by $12 \mathrm{~h}$ of incubation solely in the dark (total $24 \mathrm{~h}$ ) also in the incubator. The control group of the cells were incubated in the dark, for the exact duration of $24 \mathrm{~h}$ under identical environmental conditions except illumination. According to the assay protocol, 25 $\mu$ of the MTT reagent (Sigma-Aldrich, MO, USA) was added to each well in order to assess cell viability (final concentration: $1 \mathrm{mg} \mathrm{ml}^{-1}$ ) at the end of the $24 \mathrm{~h}$ of incubation period. Following $4 \mathrm{~h}$ of incubation of the cells with the MTT reagent, the generated formazan precipitates were solubilized by the addition of the lysing buffer $(80 \mu \mathrm{l}, \mathrm{pH}: 4.7)$, which is composed of $23 \%$ sodium dodecyl sulfate (SDS) dissolved in a solution of $45 \% N, N$-Dimethylformamide (DMF). After an overnight incubation at $37^{\circ} \mathrm{C}$, the absorbance values (of each well) were measured at $570 \mathrm{~nm}$ in a microtiter plate reader (Spectramax Plus, Molecular Devices, CA, USA) at $25^{\circ} \mathrm{C}$. Cells incubated in culture medium only (without the compound) served as the control for cell viability both for the illuminated plates and for the ones kept in the dark; whereas dimethyl sulfoxide (DMSO; $50 \%, v / v$ ) was used to observe 
maximum cell death (positive control). Cell death (\%) was assessed with the normalization of the values calculated by the formula 'optical density (OD) of control cells - OD of treated cells'. The $\mathrm{CC}_{50}$ values of the compound under illumination conditions were estimated by fitting a model with non-linear regression.

Flow cytometry. Illuminated and control K562 cells (in the dark) were stained with PE-Annexin V, as described in the technical data sheet (BioLegend, USA), and were analyzed by FACS Aria II (equipped with $488 \mathrm{~nm}$ and $635 \mathrm{~nm}$ lasers) using FACS Diva software.

Confocal laser scanning microscopy. Illuminated and control (dark) K562 cells were analyzed under a confocal laser scanning microscope (Zeiss LSM 510 META, Germany) at the excitation wavelengths of 488 or $543 \mathrm{~nm}$ to view the green (T-1) and red (PE-Annexin V) fluorescence, respectively, in order to investigate the correlation of staining of the cells with T-1 and Annexin V; operating in the sequential (multitrack) excitation/recording mode to eliminate a possible cross-talk between the channels with recording the fluorescence signal in the green (BP 505-530 nm) or red (LP 560) channel. Annexin V serves as a sensitive marker for detection of cells that are undergoing apoptosis. Images were captured at a magnification of $\times 63,1.4$ numerical aperture objective and a scan speed of $400 \mathrm{~Hz}$.

Synthesis of T-2. TFA $(0.22 \mathrm{ml}, 2.87 \mathrm{mmol})$ was added dropwise to a vigorously stirring solution of $4^{\prime}$-formyl-2,2':6',2"-Terpyridine ${ }^{29,30}(0.5 \mathrm{~g}, 1.91 \mathrm{mmol})$ and 2,4-dimethylpyrrole $(0.473 \mathrm{ml}, 4.58 \mathrm{mmol})$ in $500.0 \mathrm{ml}$ argon deaerated dichloromethane (DCM). The resulting solution was left to stir at room temperature in the dark for 1 day. $p$-Chloranil $(0.47 \mathrm{~g}, 1.91 \mathrm{mmol})$ was added in one portion and reaction was left to stir for $2 \mathrm{~h}$. Diisopropylethylamine $(8.0 \mathrm{ml})$ was added dropwise to this mixture over a period of $15 \mathrm{~min}$, and the resulting dark brown solution was allowed to stir for an additional $30 \mathrm{~min} . \mathrm{BF}_{3} . \mathrm{OEt}_{2}(8.0 \mathrm{ml})$ was then added dropwise over a period of $15 \mathrm{~min}$ and the resulting dark red solution was allowed to stir at room temperature in the dark for 1 day. The slurry reaction mixture was washed with water $(3 \times 300 \mathrm{ml})$ and dried over anhydrous $\mathrm{Na}_{2} \mathrm{SO}_{4}$. The solvent was evaporated and the residue was purified by using neutral $\mathrm{Al}_{2} \mathrm{O}_{3}$ using DCM: Hexane $(1: 1, v / v)$ as the eluent to afford compound T-2 $(0.37 \mathrm{~g}, 40.4 \%) .{ }^{1} \mathrm{H}$ nuclear magnetic resonance (NMR) $\left(400 \mathrm{MHz}, \mathrm{CDCl}_{3}\right)$ : $\delta_{\mathrm{H}} 8.70-8.73(\mathrm{~m}, 4 \mathrm{H}), 8.57(\mathrm{~s}, 2 \mathrm{H}), 7.92(\mathrm{td}, J=1.9,7.6 \mathrm{~Hz}, 2 \mathrm{H}), 7.37-7.40(\mathrm{~m}$, $2 \mathrm{H}), 6.01(\mathrm{~s}, 2 \mathrm{H}), 2.60(\mathrm{~s}, 6 \mathrm{H}), 1.56(\mathrm{~s}, 6 \mathrm{H}) .{ }^{13} \mathrm{C} \mathrm{NMR}\left(100 \mathrm{MHz}, \mathrm{CDCl}_{3}\right) \delta 156.4$, $156.1,155.3,149.4,145.3,142.8,138.8,136.9,130.5,124.3,121.6,121.1,120.6$, $30.9,15.2 \mathrm{ppm}$. High-resolution mass spectrometry (HRMS) (time-of-flight electrospray-ionization (TOF-ESI)): $m / z$ : calculated: 479.22019 , Found: $479.22179[\mathrm{M}+\mathrm{H}]^{+}, \Delta=-3.21 \mathrm{ppm}$.

Synthesis of T-1. A sample of T-2 $(0.05 \mathrm{~g}, 0.10 \mathrm{mmol})$ was dissolved in $3.0 \mathrm{ml}$ DCM. Triethyleneglycol monomethyl ether $(0.168 \mathrm{~g}, 1.0 \mathrm{mmol})$ was added to the reaction mixture which was stirred at $45^{\circ} \mathrm{C}$. The reaction was started with the addition of $\mathrm{AlCl}_{3}(0.031 \mathrm{~g}, 0.23 \mathrm{mmol})$. The progress of the reaction was followed by thin-layer chromatography (neutral $\mathrm{Al}_{2} \mathrm{O}_{3}, \mathrm{DCM}: \mathrm{MeOH}[98: 2, v / v]$ ). When all the starting material was consumed, reaction medium was cooled down to room temperature and filtered. The filtrate was concentrated under vacuum and the residue was purified by using neutral $\mathrm{Al}_{2} \mathrm{O}_{3}$ using DCM: $\mathrm{MeOH}(98: 2, v / v)$ as the eluent to afford compound T-1 $(0.068 \mathrm{~g}, 88.3 \%) .{ }^{1} \mathrm{H}$ NMR $\left(400 \mathrm{MHz}, \mathrm{CDCl}_{3}\right): \delta_{\mathrm{H}}$ $8.75-8.70(\mathrm{~m}, 4 \mathrm{H}), 8.54(\mathrm{~s}, 2 \mathrm{H}), 7.95-7.90(\mathrm{~m}, 2 \mathrm{H}), 7.41-7.37(\mathrm{~m}, 2 \mathrm{H}), 5.95(\mathrm{~s}, 2 \mathrm{H})$ $3.66-3.54(\mathrm{~m}, 2 \mathrm{H}), 3.39(\mathrm{~s}, 6 \mathrm{H}), 2.59(\mathrm{~s}, 6 \mathrm{H}), 1.52(\mathrm{~s}, 6 \mathrm{H}) .{ }^{13} \mathrm{C}$ NMR $(100 \mathrm{MHz}$, $\left.\mathrm{CDCl}_{3}\right) \delta 156.6,156.2,155.4,149.3,146.1,144.5,141.1,140.7,138.3,137.0,131.2$, $124.3,121.5,121.2,120.8,96.4,73.1,72.3,71.9,70.7,70.5,70.4,60.7,59.0,15.3$, 14.9. HRMS (TOF- ESI): $m / z$ : calculated: 789.39940 , Found: $789.40056[\mathrm{M}+\mathrm{H}]^{+}$, $\Delta=-1.47 \mathrm{ppm}$.

Data availability. The authors declare that the data supporting the findings of this study are available within the article and its Supplementary Information files.

Received: 13 June 2017 Accepted: 31 January 2018

Published online: 23 February 2018

\section{References}

1. de Silva, P. A., Gunaratne, N. H. Q. \& McCoy, C. P. A molecular photoionic AND gate based on fluorescent signalling. Nature 364, 42-44 (1993).

2. de Silva, A. P. \& Uchiyama, S. Molecular logic and computing. Nat. Nanotechnol. 2, 399-410 (2007).

3. Ozlem, S. \& Akkaya, E. U. Thinking outside the silicon box: molecular AND logic as an additional layer of selectivity in singlet oxygen generation for photodynamic therapy. J. Am. Chem. Soc. 131, 48-49 (2009).
4. Pischel, U., Andréasson, J., Gust, D. \& Pais, V. F. Information processing with molecules-quo vadis? Chemphyschem 14, 28-46 (2013).

5. Andreasson, J. \& Pischel, U. Molecules with a sense of logic: a progress report. Chem. Soc. Rev. 44, 1053-1069 (2015).

6. de Silva, A. P. Molecular logic gate arrays. Chem. Asian J. 6, 750-766 (2011)

7. Margulies, D., Melman, G. \& Shanzer, A. A molecular full-adder and fullsubtractor, an additional step toward a moleculator. J. Am. Chem. Soc. 128, 4865-4871 (2006).

8. de Ruiter, G., Tartakovsky, E., Oded, N. \& van der Boom, M. E. Sequential logic operations with surface-confined polypyridyl complexes displaying molecular random access memory features. Angew. Chem. Int. Ed. 49, 169-172 (2010)

9. Credi, A., Balzani, V., Langford, S. J. \& Stoddart, J. F. Logic operations at the molecular level. An XOR gate based on a molecular machine. J. Am. Chem. Soc. 119, 2679-2681 (1997).

10. Baytekin, H. T. \& Akkaya, E. U. A molecular NAND gate based on Watson -Crick base pairing. Org. Lett. 2, 1725-1727 (2000).

11. Ball, P. Chemistry meets computing. Nature 406, 118-120 (2000).

12. Merriam-webster.com. Definition of KILLER APP. Available at: https://www. merriam-webster.com/dictionary/killer\%20app (2017).

13. Erbas-Cakmak, S. \& Akkaya, E. U. Cascading of molecular logic gates for advanced functions: a self-reporting, activatable photosensitizer. Angew. Chem. Int. Ed. 52, 11364-11368 (2013).

14. Erbas-Cakmak, S., Cakmak, F. P., Topel, S. D., Uyar, T. B. \& Akkaya, E. U. Selective photosensitization through an AND logic response: optimization of the $\mathrm{pH}$ and glutathione response of activatable photosensitizers. Chem. Comm. 51, 12258-12261 (2015)

15. Agostinis, P. et al. Photodynamic therapy of cancer: an update. CA Cancer $J$. Clin. 61, 250-281 (2011).

16. Brown, S. B., Brown, E. A. \& Walker, I. The present and future role of photodynamic therapy in cancer treatment. Lancet Oncol. 5, 497-508 (2004).

17. Kochevar, I. E., Lynch, M. C., Zhuang, S. \& Lambert, C. R. Singlet oxygen, but not oxidizing radicals, induces apoptosis in HL-60 cells. Photochem. Photobiol. 72, 548-553 (2000)

18. Agarwal, R. et al. Apoptosis is an early event during phthalocyanine photodynamic therapy-induced ablation of chemically induced squamous papillomas in mouse skin. Photochem. Photobiol. 63, 547-552 (1996).

19. Andreasson, J. et al. A molecule-based 1: 2 digital demultiplexer. J. Phys. Chem. C 111, 14274-14278 (2007).

20. Turfan, B. \& Akkaya, E. U. Modulation of boradiazaindacene emission by cation-mediated oxidative PET. Org. Lett. 4, 2857-2859 (2002).

21. Okada, T. et al. Ultrafast intersystem crossing in some intramolecular heteroexcimers. J. Phys. Chem. 85, 3957-3960 (1981).

22. Harriman, A., Mallon, L. J., Ulrich, G. \& Ziessel, R. Rapid intersystem crossing in closely-spaced but orthogonal molecular dyads. Chemphyschem $\mathbf{8}$ 1207-1214 (2007).

23. Nagata, S., Suzuki, J., Segawa, K. \& Fujii, T. Exposure of phosphatidylserine on the cell surface. Cell Death Differ. 23, 952-961 (2016).

24. Daleke, D. L. Phospholipid flippases. J. Biol. Chem. 282, 821-825 (2007).

25. van Meer, G., Voelker, D. R. \& Feigenson, G. W. Membrane lipids: where they are and how they behave. Nat. Rev. Mol. Cell Biol. 9, 112-124 (2008)

26. Hanshaw, R. G., Lakshmi, C., Lambert, T. N., Johnson, J. R. \& Smith, B. D. Fluorescent detection of apoptotic cells by using zinc coordination complexes with a selective affinity for membrane surfaces enriched with phosphatidylserine. Chembiochem 6, 2214-2220 (2005).

27. Lakshmi, C., Hanshaw, R. G. \& Smith, B. D. Fluorophore-linked zinc(II) dipicolylamine coordination complexes as sensors for phosphatidylserinecontaining membranes. Tetrahedron 60, 11307-11315 (2004).

28. Ermolli, M., Menne, C., Pozzi, G., Serra, M. A. \& Clerici, L. A. Nickel, cobalt and chromium-induced cytotoxicity and intracellular accumulation in human hacat keratinocytes. Toxicology 159, 23-31 (2001).

29. Husson, J., Dehaudt, J. \& Guyard, L. Preparation of carboxylate derivatives of terpyridine via the furan pathway. Nat. Protoc. 9, 21-26 (2014).

30. Boechat, $\mathrm{N}$. et al. Simple reduction of heteroaromatic esters to alcohols using a sodium borohydride-methanol system. Syn. Commun. 35, 3187-3190 (2005).

31. Schmitt, A., Hinkeldey, B., Wild, M. \& Jung, G. Synthesis of the core compound of the BODIPY dye class: 4,4'-Difluoro-4-bora-(3a,4a)-diaza-sindacene. J. Fluoresc. 19, 755-758 (2009).

32. $\mathrm{Wu}, \mathrm{W}$. et al. Long-lived room-temperature near-IR phosphorescence of BODIPY in a visible-light-harvesting $\mathrm{N}^{\wedge} \mathrm{C}^{\wedge} \mathrm{N}$ PtII-acetylide complex with a directly metalated BODIPY chromophore. Chem. Eur. J. 18, 1961-1968 (2012).

\section{Acknowledgements}

We gratefully acknowledge the support from Bilkent University. 


\section{Author contributions}

I.S.T. and S.A. synthesized and characterized (chemically and spectroscopically) the target compounds; G.G. carried out the cell culture experiments; G.G. and S.A. performed microscopy experiments; E.U.A. conceived the concept and wrote the manuscript with contributions from all co-authors.

\section{Additional information}

Supplementary Information accompanies this paper at https://doi.org/10.1038/s41467018-03259-Z.

Competing interests: The authors declare no competing interests.

Reprints and permission information is available online at http://npg.nature.com/ reprintsandpermissions/

Publisher's note: Springer Nature remains neutral with regard to jurisdictional claims in published maps and institutional affiliations. (c) (i) Open Access This article is licensed under a Creative Commons Attribution 4.0 International License, which permits use, sharing, adaptation, distribution and reproduction in any medium or format, as long as you give appropriate credit to the original author(s) and the source, provide a link to the Creative Commons license, and indicate if changes were made. The images or other third party material in this article are included in the article's Creative Commons license, unless indicated otherwise in a credit line to the material. If material is not included in the article's Creative Commons license and your intended use is not permitted by statutory regulation or exceeds the permitted use, you will need to obtain permission directly from the copyright holder. To view a copy of this license, visit http://creativecommons.org/ licenses/by/4.0/.

(C) The Author(s) 2018 